\title{
Relações Amorosas, Comportamento Sexual e Vulnerabilidade de Adolescentes Afrodescendentes e Brancos em Relação ao HIV/ aids'
}

\author{
Romantic Relationships, Sexual Behavior and Vulnerability \\ of African-Descending and White Adolescents Towards HIV/
}

AIDS

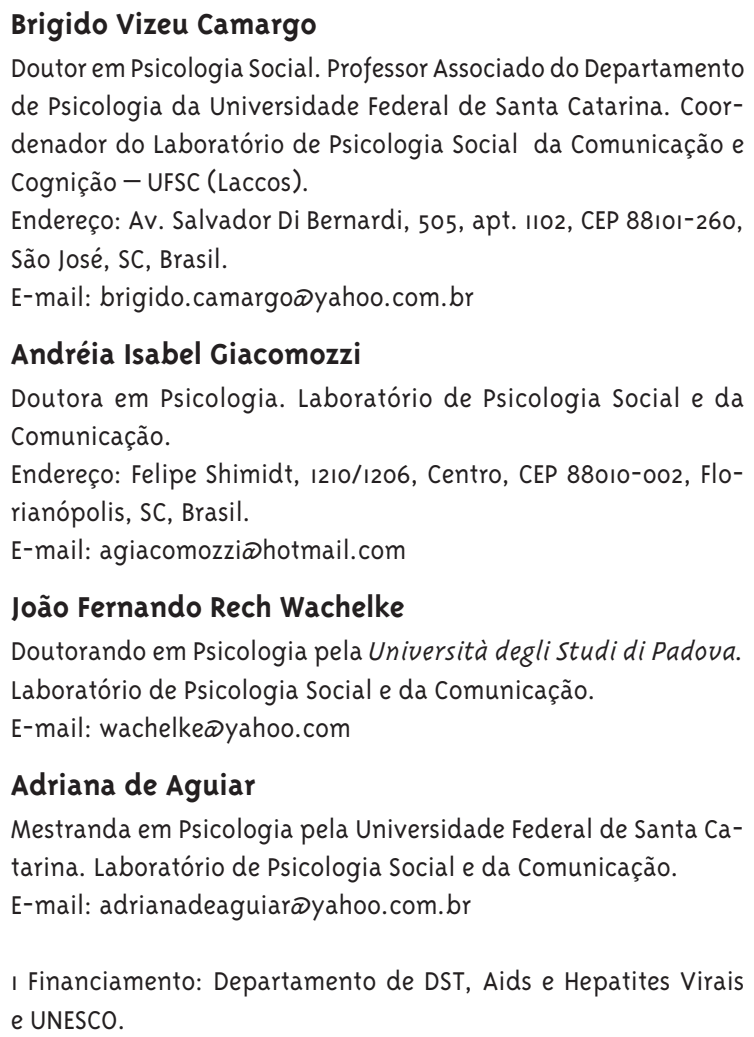

\section{Resumo}

Este estudo teve como objetivo investigar a vulnerabilidade de adolescentes brancos e afrodescendentes em relação ao HIV/aids. Para isso, investigaram-se as características socioeconômicas dos participantes e seus relacionamentos amorosos e sexuais, comportamentos de risco e prevenção, conhecimento sobre HIV/aids e atitudes frente ao uso de preservativo. $\mathrm{O}$ estudo foi realizado em regiões periféricas das cidades de Florianópolis, Itajaí e Balneário Camboriú e consistiu em duas etapas. A primeira foi qualitativa, na qual se utilizou entrevistas não diretivas com 36 estudantes de Ensino Médio, divididos igualitariamente por sexo e raça/cor. A segunda consistiu em um levantamento de dados com 715 adolescentes sobre relações afetivas e sexuais, condutas de risco e de proteção, conhecimento sobre HIV/aids e atitudes frente ao uso de preservativo. Observou-se que o nível sociocultural desfavorável associou-se aos afrodescendentes, enquanto o nível médio associou-se aos brancos. 0 conhecimento sobre aids e as atitudes frente ao uso do preservativo correlacionaram-se significativamente. Os afrodescendentes iniciam-se sexualmente mais cedo e têm mais relações amorosas esporádicas ("ficar”), porém os adolescentes brancos mantêm mais relações sexuais. Além disso, os adolescentes brancos usam menos preservativo em contexto de múltiplos parceiros e em relacionamento de namoro do que os afrodescendentes. Os resultados mostraram que, de forma geral, a maioria dos adolescentes estão vulneráveis ao HIV/aids, em 
função do baixo índice de conhecimento da doença, das práticas de risco, da importante presença do sexo desprotegido e de sua associação com as relações amorosas, como o namoro.

Palavras-chave: HIV/aids; Vulnerabilidade; Adolescência; Raça/cor; Relações amorosas.

\section{Abstract}

The present study aimed at investigating the vulnerability of white and African-descending adolescents towards HIV/AIDS. The socio-economic characteristics of participants, their romantic and sexual relationships, risk and prevention behaviors, knowledge on HIV/AIDS and attitudes towards condoms were studied for that purpose. The study was conducted in the outskirts of the cities of Florianópolis, Itajaí and Balneário Camboriú and consisted of two parts. The first one was qualitative, in which non-directive interviews with 36 secondary school students equally divided by sex and race/color were employed. The second part consisted of a survey with 715 adolescents about affective and sexual relationships, risk and protection behavior, knowledge on HIV/AIDS and attitudes towards condoms. It was observed that the unfavorable socio-cultural level was associated with the African-descending participants, while the intermediate level was linked to the whites. Knowledge on AIDS and attitudes towards condom use were significantly correlated. The African-descending begin sexual life earlier and have more episodic romantic relations ("making out"), but white adolescents have more sexual relations. Moreover white adolescents use less condoms in multiple-partner contexts and in dating relationships than the African-descending. Results indicate that in general most adolescents are vulnerable to HIV/AIDS, as a function of the low level of knowledge about the illness, risk practices, the important presence of unprotected sex and its association with romantic relationships, like dating.

Keywords: HIV/AIDS; Vulnerability; Adolescence; Race/Color; Romantic Relationships. 


\section{Introdução}

A aids é uma doença emergente, de caráter pandêmico. Por isso se apresenta, na atualidade, como um dos maiores problemas de saúde pública (Brasil, 2005). Segundo dados do Ministério da Saúde, de 1980 a 2007 , foram notificados 474.273 casos de aids no país. Segundo o Programa Conjunto das Nações Unidas sobre HIV/Aids², a aids vem apresentando taxas de incidência mais elevadas nas regiões periféricas e mais pobres, entre os trabalhadores menos qualificados e pessoas com menor grau de escolarização. Entre essas populações, os jovens estão particularmente vulneráveis por estarem no início de sua vida sexual e por apresentarem em geral comportamentos de experimentação arriscada com sentimento de invulnerabilidade. De acordo com o Boletim Epidemiológico sobre Aids (Brasil, 2007), dos 474.273 casos de aids notificados até 2007 , 10.337 referem-se a adolescentes na faixa etária dos 13 aos 19 anos.

Estudos anteriores realizados pelo LACCOS observaram algumas especificidades presentes na vulnerabilidade de estudantes do Ensino Médio diante das DST e do HIV/aids. Essas vulnerabilidades estão relacionadas à experiência de namoro (Camargo e Botelho, 2007; Bertoldo e Barbará, 2007), quantidade de parceiros sexuais (Camargo e Botelho, 2007), sexo sem proteção (Camargo e Botelho, 2007; Camargo e col., 2007; Bousfield, 2007), bem como o medo da doença (Camargo e Botelho, 2007; Camargo e col., 2007; Bousfield, 2007). Além disso, jovens de camadas populares sofrem outros agravantes, como a pobreza, a desigualdade social e a dificuldade de acesso aos serviços de saúde. Segundo Camargo e Botelho (2007), a não utilização do preservativo nas relações sexuais, a carência de informações sobre a epidemia, o desconhecimento dos riscos e a falta de programas de prevenção nos contextos escolares constituem fatores promotores do aumento de adolescentes portadores de HIV.

Estudos mostram diferenças entre homens e mulheres com relação à sexualidade e prevenção à aids (Vidal e Ribeiro, 2008; Matos e col., 2005; Antunes e col., 2002; Oliveira e col., 2005; Borges e
Schor, 2005). Em estudo realizado com adolescentes sobre a intenção do uso do preservativo, Oliveira e colaboradores (2005) observaram que a intenção comportamental de usar o preservativo sofre a influência de componentes atitudinais e normativos, tendo o primeiro componente maior peso entre os adolescentes do sexo masculino e o normativo entre as adolescentes do sexo feminino. Há um predomínio do uso do preservativo nas primeiras relações sexuais (Martins e col., 2006; Borges e Schor, 2005), porém enquanto os homens continuam a utilizá-lo nas relações subsequentes, as mulheres tendem a substituí-lo por métodos contraceptivos hormonais, como a pílula (Borges e Schor, 2005). Há também dificuldade de as mulheres negociarem o uso do preservativo com seus parceiros (Antunes e col., 2002; Giacomozzi e Camargo, 2004; Oltramari, 2004).

Embora o "ficar" seja uma forma de relacionamento bastante comum na atualidade (Matos e col., 2005), a prática de relações sexuais em contextos de relações afetivas pode se apresentar de maneira distinta para homens e mulheres. No estudo de Borges e Schor (2005), foi possível perceber que as mulheres iniciam a vida sexual predominantemente em contexto de relacionamento afetivo estável (namoro ou noivado), enquanto os homens em relações esporádicas, como as de amizade. Isso pode ocorrer, em parte, pelo fato de as mulheres relacionarem sexo e sentimentos mais estreitamente que os homens, (Vidal e Ribeiro, 2008; Matos e col., 2005). O fato de as mulheres substituírem o preservativo pela pílula e de terem suas relações sexuais em relacionamentos estáveis demonstra a influência complicadora que esse tipo de relação exerce sobre as condutas de proteção contra a aids.

Quanto ao conhecimento sobre a aids, estudos mostram a existência de relação com o contexto escolar dos estudantes (Camargo e Bertoldo, 2007; Martins e col., 2006), com o turno (Camargo e Bertoldo, 2006) e condições socioeconômicas (Ferreira, 2003). Esses estudos mostram que os estudantes de escolas públicas apresentam menor nível de conhecimento sobre a aids do que os de escola particular, principalmente entre os que frequentam o período noturno.

\footnotetext{
2 www.unaids.org.

3 Beijar e trocar carícias com parceiros sem comprometimento estável ou formal.
} 
Dados também apontam uma maior vulnerabilidade da população negra ao HIV/aids. As taxas de mortalidade por HIV/aids no estado de São Paulo em 1999, por exemplo, foram de 25,92 por 100.000 habitantes para homens negros, e de 14,44 para brancos. Dentre as mulheres, as taxas foram de 11,39 e 4,92, respectivamente para negras e brancas. A diferença da taxa de mortalidade entre mulheres negras e brancas indica que as mulheres negras morrem 2,3 vezes mais que as brancas em virtude de HIV/aids, enquanto os homens negros morrem 1,7 vez mais que os brancos (Batista, 2005). Esses dados corroboram os resultados encontrados por Lopes e colaboradores (2007) em estudo sobre vulnerabilidade ao HIV/aids em mulheres negras e brancas portadoras do vírus no município de São Paulo. Neste estudo, as mulheres negras apresentaram: menor nível de escolaridade e renda do que as brancas, menor taxa de atendimento por outros profissionais de saúde além do médico infectologista e, menor nível de conhecimento correto sobre o exame $\mathrm{CD}_{4}$ e carga viral, quando comparadas às mulheres brancas.

Segundo Ayres e colaboradores (1999) a vulnerabilidade individual envolve três pressupostos: a) qualquer indivíduo é passível de contaminação em determinada época da vida; b) quanto maior o amparo social e a assistência à saúde de que os indivíduos dispuserem menor a morbidade, invalidez e morte; c) questões de ordem cognitiva, comportamental e social afetam diretamente a vulnerabilidade individual. Assim a vulnerabilidade tem relação com o conhecimento que se tem da epidemia. Para se compreender esse tipo de conhecimento e sua importância para a vulnerabilidade dos jovens afrodescendentes e brancos, torna-se essencial o estudo das suas crenças, informações e atitudes sobre a doença, bem como suas condutas protetoras e arriscadas (práticas sexuais vulneráveis no contexto das relações amorosas e sexuais, etc.).

0 conceito de atitudes pretende ser mediador entre a forma de pensar e a forma de agir dos indivíduos, pois tem ligação com os comportamentos (Vala, 200o), permitindo identificar o posicionamento de um indivíduo diante da realidade social. As atitudes envolvem o que as pessoas pensam, sentem e como gostariam de se comportar em relação à determinado objeto. Essa relação entre os componentes de uma atitude pode ser de coerência ou incoerência, pois é possível encontrar inconsistência entre as atitudes e os comportamentos expressos pelas pessoas. (Rodrigues e col., 1996). Dessa forma, os fatores associados ao risco em relação à aids vão além do conhecimento sobre a prática de sexo seguro e o conhecimento de risco, pois no momento em que o indivíduo necessita tomar uma decisão diante de uma situação, os estados emocional e cognitivo podem influenciar sua avaliação de riscos e, consequentemente, sua decisão no uso do preservativo (Foreman, 2003; Gutnik e col., 2006). Observa-se que a atitude de jovens a respeito do uso do preservativo em geral é positiva (Camargo e Botelho, 2007; Camargo e Bertoldo 2006; Martins e col., 2006), porém, nem sempre há coerência entre essa atitude e o efetivo comportamento de seu uso.

A psicologia social tem contribuído para a compreensão da relação entre o conhecimento e as práticas preventivas em saúde. Godin (1991) descreve as principais teorias explicativas da adoção de determinado comportamento por parte dos indivíduos no que se refere a esse domínio. Segundo ele, para o modelo das "crenças sobre a saúde" (health belief model, de Becker), a percepção de uma ameaça é que explica o comportamento preventivo; já para o modelo da ação refletida de Fishbein e Ajzen, é a intenção composta de atitudes sobre o comportamento e pelas normas subjetivas (oriundas da relação com pessoas influentes para o indivíduo) que conduz à adoção do comportamento; para o modelo dos comportamentos interpessoais de Triandis, é uma complementaridade entre a intenção, o hábito e as condições facilitadoras ou complicadoras da conduta que operam; e, por último, as novas contribuições da teoria do comportamento planejado de Ajzen e Madlen acrescentam a percepção que o indivíduo tem do seu controle sobre o comportamento como uma variável importante para entender a relações entre atitudes e comportamentos.

A partir dessas constatações, o objetivo deste estudo foi investigar a vulnerabilidade de estudantes afrodescendentes e brancos à infecção pelo HIV/aids, por meio de algumas variáveis psicossociais associadas à suscetibilidade dos adolescentes à epidemia. Embora o estudo tenha uma intenção descritiva, também é comparativo, no que se refere à variável 
raça/cor. As variáveis psicossociais consideradas por este estudo são as características das relações amorosas e sexuais, o conhecimento sobre a aids, a atitude sobre a utilização do preservativo e comportamentos de proteção e de risco diante do HIV.

Esta pesquisa foi realizada em duas etapas, a primeira qualitativa com entrevistas, e a segunda quantitativa com questionários. Teve caráter transversal, com amostragem não randômica. A coleta das duas etapas foi realizada com estudantes dos períodos noturnos de escolas públicas de bairros periféricos das cidades de Florianópolis, Itajaí e Balneário Camboriú, principais cidades em termos de incidência do HIV/aids do estado de Santa Catarina. Optou-se pelo período noturno e escolas de periferia, pois buscavam-se adolescentes trabalhadores que residissem em regiões menos valorizadas comercialmente, o que caracterizaria adolescentes com situação socioeconômica mais desfavorável. A caracterização dos adolescentes quanto à raça/cor obedeceu ao critério de autodeclaração. Sugeriam-se as seguintes opções: "branco", "negro”, "mulato”, "pardo" ou "outra raça/cor". Os estudantes que se autodeclararam negros, mulatos e pardos, foram considerados afrodescendentes para este estudo.

\section{Primeira Etapa de Pesquisa}

Participaram dessa etapa da pesquisa 36 estudantes de Ensino Médio na faixa etária de 15 a 24 anos. Foram 12 estudantes de cada cidade, divididos igualmente por sexo e raça/cor. A primeira etapa teve objetivo exploratório, seus resultados subsidiaram a construção do instrumento utilizado na segunda etapa.

Foi utilizada a entrevista não diretiva em profundidade (Ghiglione e Matalon, 1993). Para a cobertura dos tópicos focalizados, os entrevistadores dispunham de uma lista de pontos a serem explorados: amizade, namoro e relações afetivas, relações sexuais, contracepção, uso de preservativos e métodos contraceptivos, doenças sexualmente transmissíveis e aids. As entrevistas foram realizadas individualmente, em local reservado, nas escolas dos estudantes, com o consentimento do corpo docente e dos entrevistados, com duração aproximada de 45 minutos cada.

\section{Resultados Primeira Etapa}

As entrevistas foram transcritas e analisadas com o auxílio do software Alceste (Analyse Lexicale par Contexte d'un Ensemble de Segments de Texte) (Reinert, 1990; Camargo, 2005), que emprega uma análise hierárquica descendente, permitindo uma análise lexicográfica do texto e a formação de contextos caracterizados por vocabulário e segmento de textos similares (classes). O conjunto de entrevistas transcritas compôs o corpus de análise, que apresentou 82.593 ocorrências, com 4.860 palavras diferentes. Aproximadamente, $41 \%$ das ocorrências foram analisadas, com frequências iguais ou superiores a 4. O número total de palavras com frequência igual a 1, um índice de heterogeneidade do vocabulário empregado pelos participantes, foi de 2.171.

0 corpus analisado foi composto de 36 Unidades de Contexto Inicial (UCIs), correspondentes às 36 entrevistas realizadas. 0 programa subdividiu as UCIs em 1.763 Unidades de Contexto Elementar (UCEs) ou segmentos de texto. A classificação hierárquica descendente (CHD) considerou 1.219 UCEs, representando $69,14 \%$ do total de UCEs. A figura 1 mostra as relações entre as 7 classes resultantes da CHD.

Foram encontradas diferenças nos conteúdos representacionais quanto a sexo e raça/cor. No que se diz respeito à aids, as meninas enfatizaram dois pontos: proximidade com a doença (relações familiares e com pessoas de sua rede social que possuem a doença) e realização do exame para detecção do HIV. A classe 1 (proximidade com a aids) aparece relacionada com as meninas afrodescendentes, o que sugere que o contato com a doença está mais presente em seus cotidianos. Os meninos falaram mais a respeito de seu conhecimento sobre a aids (formas de contágio e consequências da doença). A maior associação das meninas com as classes sobre contracepção, preservativo e gravidez demonstra uma maior preocupação delas com esses conteúdos. As meninas relacionam o preservativo à prevenção da aids, mas encontram barreiras na negociação da sua utilização com seus parceiros, pois, segundo elas, os meninos relatam não gostar de usar preservativo e se referem ao não uso como uma "prova de confiança/amor". O uso de outros métodos anticoncepcionais, como a pílula, também contribui para a prática do sexo sem proteção. Os participantes do sexo masculino associaram-se às 


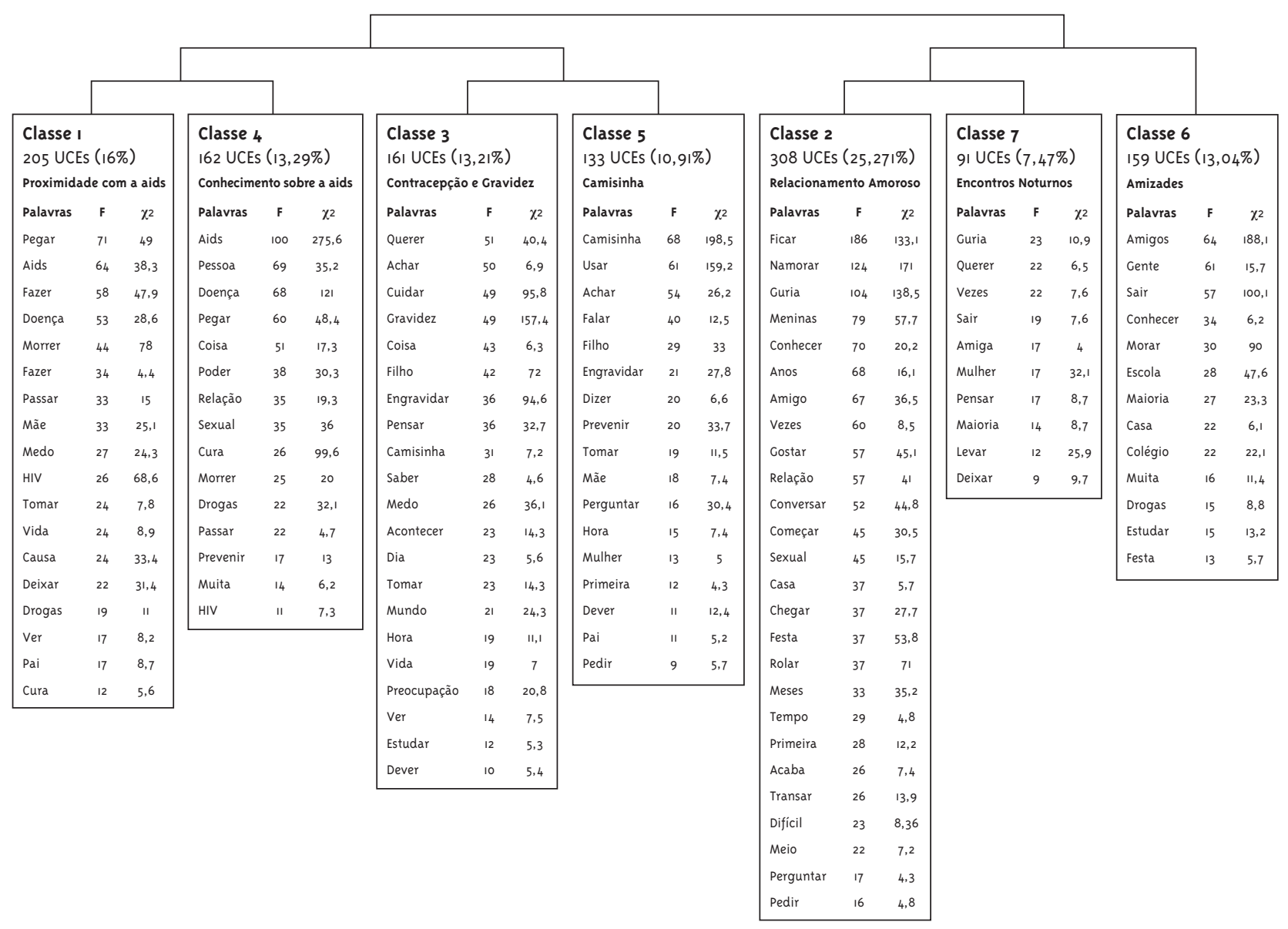

classes sobre encontros noturnos e relacionamentos amorosos com as garotas (namoro, ficar, primeiras experiências sexuais), estando a primeira mais relacionada aos participantes afrodescendentes e a segunda aos participantes brancos.

\section{Segunda Etapa da Pesquisa}

A partir dos resultados da primeira etapa, realizou-se um estudo quantitativo, de natureza descritiva e comparativa. Para isso, foi utilizado um questionário semiestruturado e autoadministrado, elaborado a partir das indicações do estudo anterior. Os questionários foram analisados com auxílio do software SPSS 11.o (SPSS, 2001).

Realizou-se uma categorização do nível sociocultural dos participantes conforme critério já utilizado por Camargo (1997): foi solicitado aos participantes que indicassem a profissão e o nível de escolaridade de seus pais. Foram considerados de nível sociocultural Desfavorável os filhos de pais com até o Ensino Médio incompleto, com profissões simples, como operários e prestadores de serviços operacionais; filhos de pais operários, funcionários ou profissionais liberais com pelo menos o Ensino Médio completo, foram classificados de nível sociocultural Médio e filhos de funcionários ou profissionais liberais com Ensino Superior foram classificados de nível sociocultural Favorável.

\section{Resultados da Segunda Etapa}

Conforme a tabela 1, o delineamento empregado buscou equilibrar o número de participantes segundo a cidade de residência, o sexo e a raça/cor. 
Tabela I - Delineamento da segunda etapa

\begin{tabular}{|c|c|c|c|c|}
\hline \multirow{2}{*}{ Cidade } & \multirow{2}{*}{ Etnia } & \multicolumn{2}{|c|}{ Sexo } & \multirow[b]{2}{*}{ Total } \\
\hline & & Masculino & Feminino & \\
\hline \multirow[t]{3}{*}{ Florianópolis } & Branco & 50 & 70 & 120 \\
\hline & Afrodescendente & 53 & 67 & 120 \\
\hline & Total & 103 & 137 & 240 \\
\hline \multirow[t]{3}{*}{ Itajaí } & Branco & 62 & 58 & 120 \\
\hline & Afrodescendente & 79 & 41 & 120 \\
\hline & Total & 141 & 99 & 240 \\
\hline \multirow[t]{4}{*}{ Bal. Camb. } & Branco & 61 & 59 & 120 \\
\hline & Afrodescendente & 62 & 53 & 115 \\
\hline & Total & 123 & 112 & 235 \\
\hline & Total geral & 367 & 348 & 715 \\
\hline
\end{tabular}

Com relação à raça/cor, 360 (50,3\%) declararam-se brancos, 135 (18,9\%) declararam-se mulatos, 130 $(18,2 \%)$ declararam-se pardos e $90(12,6 \%)$ declararam-se negros. A média de idades dos participantes foi de 17 anos e três meses ( $\mathrm{dp}=2$ anos); 83,6\% dos participantes já haviam tido uma experiência de trabalho. 0 trabalho adolescente indica a necessidade econômica, reforçando a vulnerabilidade dos participantes da pesquisa. Em relação ao nível sociocultural, observou-se, que $80,2 \%$ dos participantes apresentam nível desfavorável, e houve associação significativa entre a raça/cor dos participantes (branco ou afrodescendente) e seu nível sociocultural [ $\chi_{2}=$ $7,21 ; \mathrm{gl}=2 ; \mathrm{p}<0,05]$, indicando que há, proporcionalmente, mais afrodescendentes com nível sociocultural desfavorável que brancos, e mais brancos com nível sociocultural médio que afrodescendentes. A proximidade dos adolescentes, participantes desta pesquisa, em relação à aids e ao HIV é importante: 22,8\% deles disseram conhecer um amigo com a doença e 15,5\% declararam ter um familiar nessa condição, porém não houve diferenças estatisticamente significativas com a variável raça/cor.

\section{Relações Afetivas, Sexuais e Conduta Protetora}

Mais de dois terços dos participantes da pesquisa declararam que costumam "ficar", sendo esse comportamento mais frequente entre os afrodescenden- tes $(73,9 \%)$ do que entre os brancos $(63,3 \%)$. Houve diferença estatisticamente significativa entre a variável "ficar" e a raça/cor $\left[\chi^{2}=8,61 ; \mathrm{gl}=1 ; \mathrm{p}<0,01\right]$. Em relação à variável namoro não houve diferenças estatisticamente significativas entre brancos e afrodescendentes, porém, a duração do namoro é maior entre os primeiros, embora a diferença não seja estatisticamente significativa $\left[\chi^{2}=6,09 ; \mathrm{gl}=3\right.$; NS]

Considerando-se a variável raça/cor, observou-se que tanto para brancos quanto para afrodescendentes ter um namorado(a) implica na diminuição das relações eventuais de "ficar". Para os brancos, entre os que afirmaram ter namorado(a) 6o,6\% afirmaram não ter o hábito de "ficar", enquanto esse percentual subiu para 83,2\% entre os que afirmaram não ter namorado(a) $\left[\chi^{2}=71,54 ; \mathrm{gl}=1 ; \mathrm{p}<\mathrm{o}, \mathrm{oo} 1\right]$. Para os afrodescendentes, entre os que afirmaram ter namorado(a), 54,1\% declararam que costumam "ficar" mesmo assim, e esse percentual subiu para $86,5 \%$ entre os que dizem não ter namorado(a) $\left[\chi^{2}=\right.$ 44,$16 ; \mathrm{gl}=1 ; \mathrm{p}<\mathrm{o}, \mathrm{oo} 1$ ]

A média de idade de toda a amostra para a primeira relação sexual foi de 14 anos e nove meses ( $d p=1$ ano e nove meses). Com relação à raça/cor, os afrodescendentes declararam que tiveram a primeira relação sexual mais cedo do que os brancos, pois a média para os brancos foi de 14 anos e 11 meses ( $d p=$ 1 ano e 7 meses) e para os afrodescendentes, 14 anos e 6 meses ( $d p=2$ anos). A diferença foi estatisticamente significativa $[\mathrm{F}(1,521)=7,57 ; \mathrm{p}<0,05]$. 
Quanto à frequência de experiências sexuais entre as pessoas que disseram "ficar", a figura 2 mostra uma distribuição semelhante para brancos e afrodescendentes. Os participantes concentraram-se na modalidade que relatou ter tido relações sexuais uma ou algumas vezes, seguidos pelos participantes que disseram ter tido muitas relações sexuais.

Entre os indivíduos que não costumavam "ficar", há diferenças quanto à raça/cor. Os brancos decla- raram predominantemente ter tido relações sexuais muitas vezes, seguidos em proporção pelos que nunca tiveram relações e que tiveram relações algumas vezes, com proporções próximas $\left[\chi^{2}=11,09 ; \mathrm{gl}=2 ; \mathrm{p}\right.$ $<0,01$; V Cramer $=0,18]$. Entre os afrodescendentes, houve predomínio dos que disseram tê-las tido até algumas vezes, seguidos daqueles que declararam nunca haver tido relações sexuais $\left[\chi^{2}=22,47 ; \mathrm{gl}=2\right.$; $\mathrm{p}<\mathrm{o}$, oo1; V Cramer $=0,26$ ].

\section{Figura 2 - Relação entre o comportamento de "ficar" e frequência de relações sexuais em função de raça/cor}

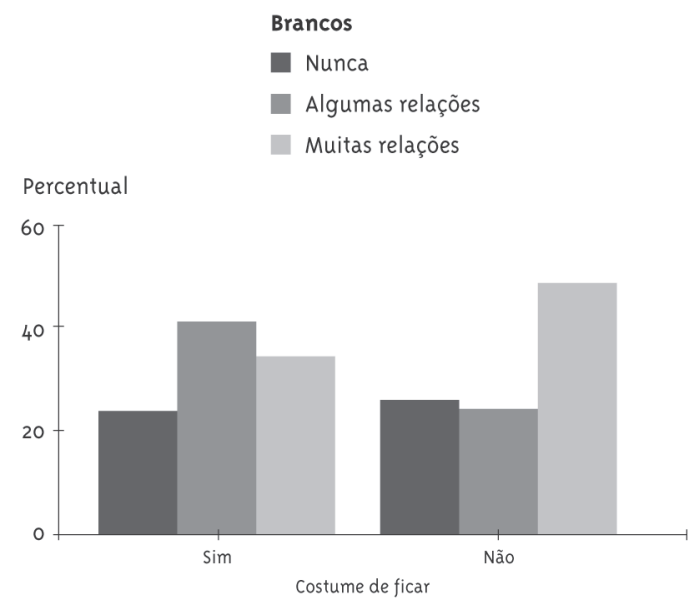

Entre os participantes que disseram ter o costume de "ficar", 6o,4\% declararam ter usado o preservativo em todas as relações sexuais. Para os participantes que não "ficavam", predominou o comportamento de ter utilizado o preservativo somente em algumas relações (45,8\%). Houve associação significativa entre ter o costume de "ficar" e utilizar o preservativo. $\left[\chi^{2}=25,76\right.$; gl =2; $p$ $<$ o,oo1]. Comparando brancos e afrodescendentes, os resultados distribuíram-se conforme observado na amostra geral: as pessoas que "ficavam", fossem brancos ou afrodescendentes, utilizavam mais sistematicamente o preservativo nas suas relações sexuais, em comparação às pessoas que não "ficavam" (ver figura 3).

Observou-se, porém, na figura 3, que entre brancos que não tinham o costume de "ficar" a proporção dos que não usaram preservativo foi maior do que entre os que ficavam (23,3\% contra $13 \%)$. Entre os afrodescendentes, esse aumento não chegou a um

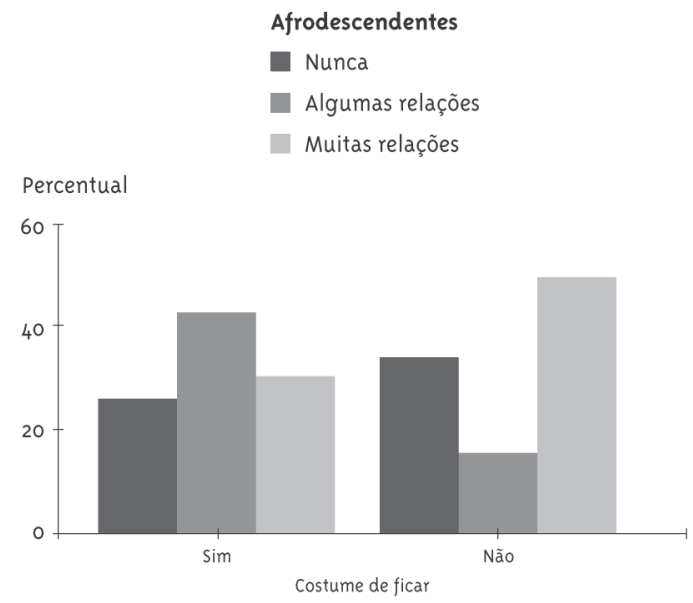

ponto percentual. Foram observadas associações significativas entre a prática amorosa de "ficar" e o uso do preservativo, tanto para brancos $\left[\chi^{2}=15,14\right.$; $\mathrm{gl}=2 ; \mathrm{p}<\mathrm{o}, 01]$ quanto para afrodescendentes $\left[\chi^{2}=\right.$ 11,36; $\mathrm{gl}=2 ; \mathrm{p}<\mathrm{o}, \mathrm{o1}$ ].

De forma inversa ao comportamento de "ficar", entre os participantes que namoravam a maior proporção declarou que usava preservativo apenas em algumas relações (43,1\%), embora 41,3\% declararam ter usado preservativo em todas as relações sexuais. Para os que não namoravam o uso preservativo em todas as relações sexuais foi declarado pela maioria $(68,5 \%)$, diminuindo consideravelmente a proporção de participantes que declararam usá-lo apenas em algumas relações (20\%).

A figura 4 mostra que entre os brancos que namoravam quase metade dos participantes usaram preservativo somente em algumas relações, enquanto uma proporção menor declarou usar preservativo em todas as relações sexuais. Entre os brancos que 
Figura 3 - Relação entre comportamento de "ficar" e uso do preservativo em função de raça/cor

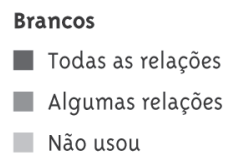

Percentual

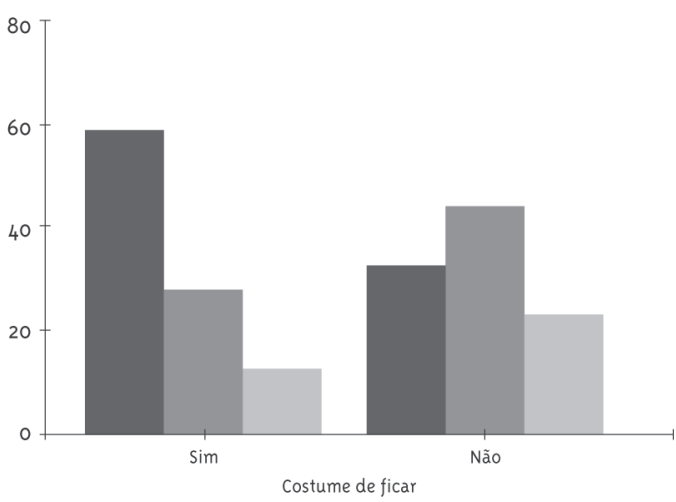

Afrodescendentes

- Todas as relações

Algumas relações

Não usou

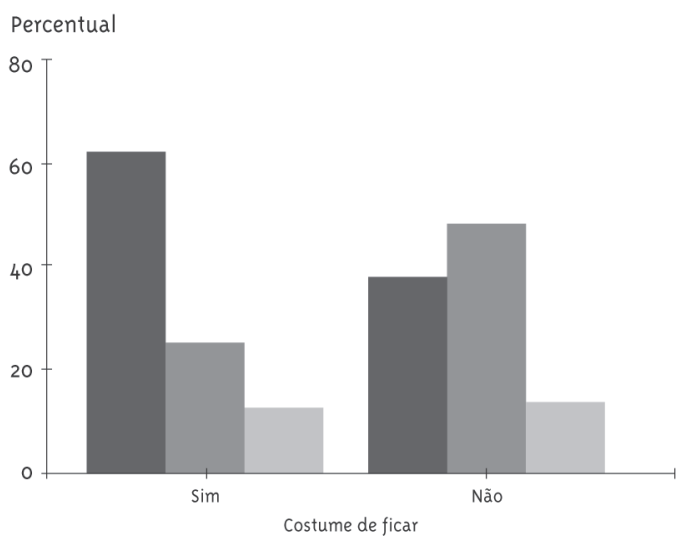

Figura 4 - Relação entre namoro e uso do preservativo em função de raça/cor

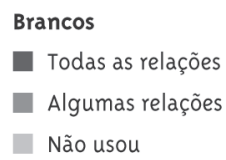

Todas as relações

Algumas relações

Não usou

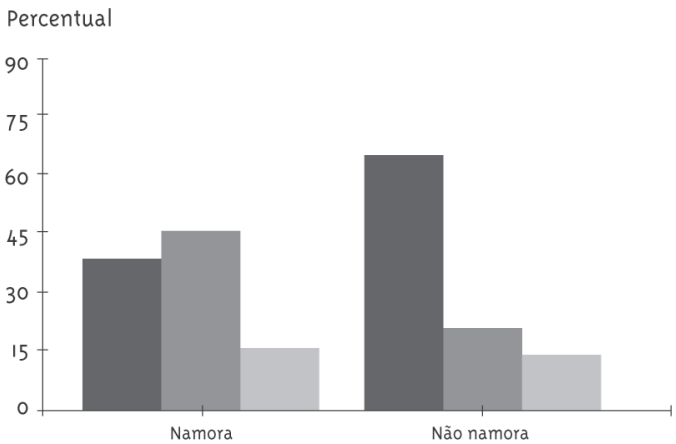

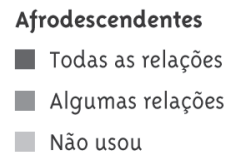

Percentual

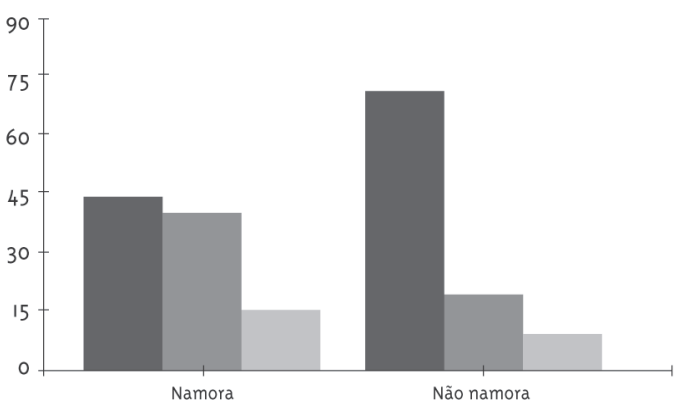

não namoravam houve maior proporção de respondentes que disseram se proteger: $65,3 \%$ declararam ter usado preservativo em todas as relações e apenas $20,8 \%$ somente em algumas relações.

Entre os afrodescendentes que namoravam, a proporção predominante de participantes, diversamente, declarou ter usado o preservativo em todas as relações, enquanto entre os que não namoravam, verificou-se uma proporção ainda maior do que entre os brancos, que declarou ter usado sistematicamente o preservativo nas relações sexuais. Foram verificadas associações estatisticamente significativas entre ter namorado e usar de forma não sistemática o preservativo, tanto para brancos $\left[\chi^{2}=17,49 ; \mathrm{gl}=2\right.$; $\mathrm{p}<\mathrm{o}$, oo1 $]$ quanto para afrodescendentes $\left[\chi^{2}=15,31\right.$; $\mathrm{gl}=2 ; \mathrm{p}<\mathrm{o}, \mathrm{ool}]$. 
Figura 5 - Relação entre múltiplos parceiros e uso sistemático do preservativo em função de raça/cor

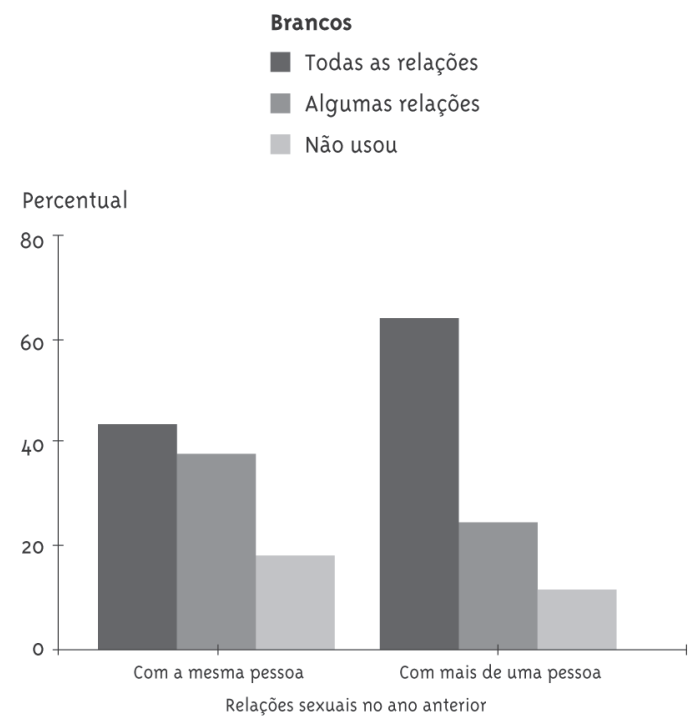

A respeito da associação entre quantidade de parceiros sexuais e o uso do preservativo, considerando a variável raça/cor como controle, os resultados indicam que os afrodescendentes apresentam uma proporção menor de participantes que não usaram o preservativo no contexto de múltiplos parceiros do que aquela apresentada pelos brancos (respectivamente 3,1\% e 11,5\%) (ver Figura 5). Houve associação estatisticamente significativa entre quantidade de parceiros sexuais e uso do preservativo para brancos $\left[\chi^{2}=7,42 ; \mathrm{gl}=2 ; \mathrm{p}<0,05\right]$ e igualmente para afrodescendentes $\left[\chi^{2}=9,39 ; \mathrm{gl}=2 ; \mathrm{p}<0,01\right]$.

\section{Teste HIV e Uso do Preservativo}

A maioria dos participantes $(80,1 \%)$ disse nunca ter realizado o teste para detecção do vírus HIV. Não houve associação estatisticamente significativa entre raça/cor e o fato de realizar esse tipo de teste.

Os participantes que não usaram preservativos foram os que mais disseram ter feito o teste (43,8\%), enquanto os que usaram preservativo em algumas relações relataram uma proporção menor de realização do teste (32,6\%). Assim, foi observada associação estatisticamente significativa entre o uso de preservativo nas relações sexuais e o fato de não ter realizado teste de HIV $\left[\chi^{2}=14,41 ; \mathrm{gl}=2 ; \mathrm{p}<\mathrm{O}, 01\right]$. Mas isso
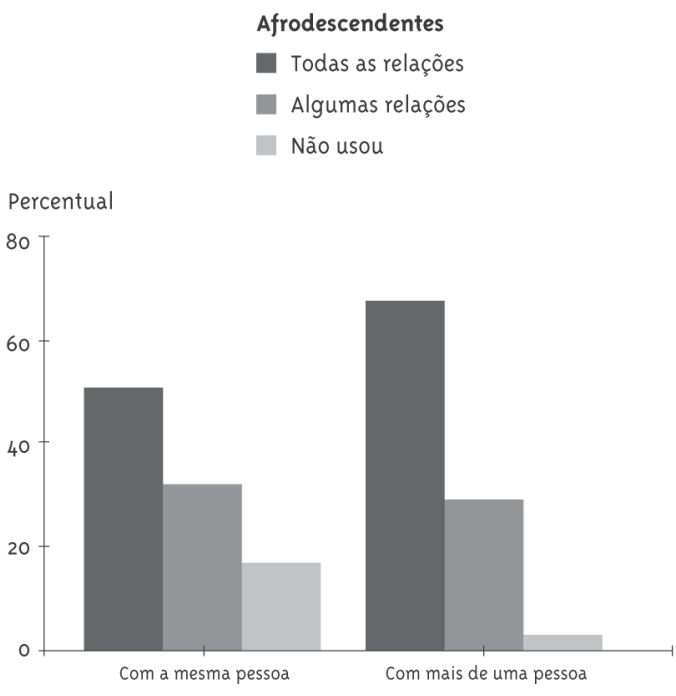

Relações sexuais no ano anterior

se circunscreve aos participantes brancos $\left[\chi^{2}=10,81\right.$; $\mathrm{gl}=2 ; \mathrm{p}<\mathrm{o}, \mathrm{ol}]$, pois entre os afrodescendentes não foi encontrada associação significativa entre essas duas variáveis $\left[\chi^{2}=4,10 ; \mathrm{gl}=2 ; \mathrm{NS}\right]$. Os brancos que não usaram preservativos foram os que em maior número fizeram o teste para detecção do $\operatorname{HIV}(47,4 \%$ dos brancos e $38,5 \%$ dos afrodescendentes).

\section{Conhecimento sobre aids e Atitude Quanto ao Uso do Preservativo}

No que se diz respeito ao conhecimento sobre a aids, não houve diferenças estatisticamente significativas nos escores obtidos no subteste usado para medir conhecimento sobre HIV/aids (Camargo e col., 2005). Os participantes brancos apresentaram uma média de 6,42 acertos (do total de 10 itens), enquanto os afrodescendentes tiveram uma média de 6,22 acertos. No entanto, o ponto de corte para se considerar o respondente bem informado sobre HIV/aids é 7 itens. Verificou-se também uma relação entre as variáveis "conhecimento sobre a aids" e "namoro" em relação à variável "raça/cor", indicando que os adolescentes afrodescendentes que namoravam possuíam um maior escore no teste de conhecimento sobre aids do que os que não namoravam $[F(2,336)$ $=4,25 ; p=0,05 ; d=0,09]$. 
Com relação aos posicionamentos sobre a utilização do preservativo, ambos os grupos (brancos e afrodescendentes) apresentaram atitudes favoráveis. Porém, observou-se que as meninas têm uma atitude mais favorável ao uso do preservativo que os meninos, $[F(1,711)=35,94 ; p<0,001]$, com efeito considerado médio da variável "sexo" $(\mathrm{d}=\mathrm{o}, 44)$. Observou-se também que a maior favorabilidade ao uso do preservativo está associada ao comportamento de seu uso sistemático nos últimos 12 meses, tanto para os adolescentes brancos $[\mathrm{F}(2,234)=5,20 ; \mathrm{p}<$ $\mathrm{o}, \mathrm{o} 1 ; \mathrm{d}=0,42]$ quanto para afrodescendentes $[\mathrm{F}(2$, 208) = 3,13; $\mathrm{p}=\mathrm{o}, 05 ; \mathrm{d}=\mathrm{o}, 34 \mathrm{l}$.

O conhecimento sobre aids e a atitude diante do uso do preservativo correlacionaram-se significativamente, mas com intensidade de pequena magnitude $(\mathrm{r}=0,09 ; \mathrm{p}<0,05)$. Foi observado também que as pessoas sem experiência sexual tiveram atitudes mais favoráveis ao uso do preservativo, isto é, uma média maior na escala $(3,81 ; d p=0,44)$ que os participantes que tiveram até algumas (média 3,59 e $d p=0,50$ ) ou muitas relações sexuais (média $3,64$ e dp $=0,54)$.

\section{Discussão}

Este estudo procurou identificar as variáveis intervenientes nas vulnerabilidades de jovens brancos e afrodescendentes com relação às DST/HIV/aids. Os resultados apontaram uma diferença com relação ao nível sociocultural, uma vez que mesmo que a pesquisa tenha sido feita com estudantes de escolas públicas de bairros periféricos e do período noturno, os participantes afrodescendentes foram maioria a ser classificados em nível sociocultural desfavorável enquanto a maioria dos brancos foi classificada no nível sociocultural médio. Isso acontece porque no Brasil, a raça/cor está frequentemente associada à classe social, em que uma proporção importante de afrodescendentes pertence aos estratos socioeconômicos mais carentes.

Constatou-se ainda que os adolescentes brancos e afrodescendentes apresentam diferenças em suas relações amorosas e sexuais. Os afrodescendentes iniciam-se sexualmente mais cedo e têm mais relações amorosas esporádicas ("ficar”), porém os adolescentes brancos mantêm mais relações sexuais.
Além disso, os adolescentes brancos usam menos preservativo em contexto de múltiplos parceiros e em relacionamento de namoro do que os afrodescendentes.

Tanto para brancos como para afrodescendentes, uma das variáveis mais associadas à relação sexual sem preservativo é o namoro. 0 fato de estar em uma relação estável e a presença de confiança entre o casal diminui o uso do preservativo, proporcionando um sentimento de invulnerabilidade, indo ao encontro dos achados de estudos anteriores (Camargo e Botelho, 2007; Vinaccia e col., 2007; Griep e col., 2005; Giacomozzi, 2005; Giacomozzi e Camargo, 2004; Oltramari e Camargo, 2004). Além disso, o uso de outros métodos anticoncepcionais, como a pílula, também contribui para a prática do sexo sem proteção. Corroborando o estudo de Camargo e Botelho (2007), na presente pesquisa as meninas também se mostraram mais favoráveis ao preservativo do que os meninos, porém, na primeira etapa da pesquisa observou-se que elas sentem dificuldade em negociar o uso do preservativo com seus parceiros sexuais. A desigualdade de poder nas relações entre homens e mulheres é um dos motivos da dificuldade que ambos têm em discutir formas seguras de exercer a sexualidade (Villela, 1998), expondo-os por consequência às doenças sexualmente transmissíveis (DST) e à aids.

Observa-se influência das questões de gênero nos comportamentos de risco diante do HIV. Estas questões, somadas às culturais e econômicas, têm grande peso no comportamento sexual da mulher e evidentemente influem nas questões de poder e de tomada de decisões: “... especialistas em gênero e comportamento sexual notam que gênero é obviamente importante para definir como serão as negociações nos encontros sexuais e quem determinará quais práticas irão prevalecer" (Ehrhardt e Wasserheit, 1991, p. 99).

Além disso, neste estudo, tanto para brancos como para afrodescendentes, o uso ou não do preservativo está associado às variáveis psicossociais: a experiência afetiva do namoro e a atitude ou o posicionamento do adolescente em relação ao preservativo. A primeira apareceu como fator complicador para a adoção da conduta do uso sistemático do preservativo, já a segunda parece exercer um 
papel inverso, como um fator facilitador. No entanto, entre os afrodescendentes a variável psicossocial "conhecimento" também aparece associada à adoção de conduta protetora. Entre estes um maior conhecimento sobre aids apresentou-se correlacionado a uma atitude mais favorável ao uso do preservativo e os que namoravam tinham um melhor nível de conhecimento sobre aids. Porém constatou-se que apenas o conhecimento sobre a epidemia não é suficiente para a adoção de condutas protetoras, uma vez que o uso do preservativo é menos frequente entre os que vivenciam um relacionamento afetivo estável.

Segundo Jodelet (1998), a recusa do uso do preservativo em relações afetivas estáveis pode ter como uma das explicações para o temor de a pessoa manifestar sinais de desconfiança para com o parceiro; pode representar uma medida que quebra o encanto durante o ato de amor, contradizendo o caráter natural e apaixonado existente durante as relações íntimas. O significado simbólico do preservativo varia no contexto de cada relacionamento. A proposição do uso do preservativo, dependendo do objetivo, pode então significar desconfiança ou infidelidade para alguns e cuidado e amor para outros; sexo desprotegido pode significar irresponsabilidade dentro de um relacionamento e compromisso em outro (Van Campenhoudte col., 1997). Em relacionamentos estáveis, a principal preocupação é com a gravidez, portanto o uso do preservativo com o objetivo de preveni-la adquire valor positivo. A discussão do HIV nestes tipos de relacionamentos pode assumir um significado negativo, pois pode materializar a certeza da infidelidade do parceiro (Madureira e Trentini, 2008). Em contextos de relacionamentos casuais, como o "ficar", a preocupação com as doenças é socialmente bem-aceita e incentivada, facilitando a adoção de estratégias preventivas.

Com relação à realização do teste anti-HIV, observou-se que aqueles que passaram pelo teste foram os que utilizaram menos o preservativo, ou seja, o teste foi procurado pelos que sentiram algum risco de ter contraído a doença. Estes dados são corroborados por análises de dados de testes anti-HIV feitos nos CTA de Florianópolis. No ano de 2009, 44,4\% das pessoas que realizaram o teste declararam que o estavam fazendo porque tiveram comportamento de risco (Prefeitura Municipal de Florianópolis, 2009).

\section{Considerações Finais}

Observou-se neste estudo a capacidade preditiva das atitudes diante do uso do preservativo sobre o comportamento de utilizá-lo. Sabe-se que quanto maior for o interesse investido pelo indivíduo no conteúdo atitudinal, maior será a correspondência entre as atitudes e o comportamento, aumentando assim a capacidade preditiva de comportamentos pelas atitudes (Budd, 1986; Parker e col., 1974), maior também será a sua resistência a mudanças (Gorn, 1975; Zuwerink e Devine, 1996), e mais poderosamente se ligará/influenciará as atitudes de outras pessoas sobre o mesmo objeto (Krosnick, 1988; McGraw e col., 1990).

As atitudes são construídas através da interação social, produto de comparações, sucessivas identificações e diferenciações que permitem situar uma posição sobre um objeto diante dos outros em dado momento. São também passíveis de mudança e transformação através da informação ou da experiência (Rodrigues e col., 2002).

No caso específico dos afrodescendentes, observou-se a associação das atitudes com o nível de conhecimento sobre aids. Isso acontece, pois, de acordo com o modelo de Rosenberg e Hovland (1960), as atitudes envolvem um sistema composto de três dimensões: cognitiva, afetiva e comportamental. E a dimensão cognitiva (conhecimento) mostrou-se intimamente relacionada ao comportamento do uso do preservativo.

Pode-se apontar como um limite deste estudo a troca do sistema classificatório da variável raça/cor, uma vez que os participantes que se autodeclararam negros, mulatos e pardos foram agrupados pelos pesquisadores em afrodescendentes para efeitos comparativos. Porém, de uma forma geral, a maioria desses adolescentes, afrodescendentes ou não, estão vulneráveis ao HIV/aids, em função do baixo nível de conhecimento sobre a doença, da importante presença do sexo desprotegido e da sua associação com as relações amorosas (principalmente o namoro), e da proximidade com pessoas infectadas pelo HIV. Certamente há outros fatores, não relatados 
aqui, que podem estar associados à vulnerabilidade desses adolescentes diante do HIV, como a exposição à violência e uso abusivo de drogas.

A composição desses fatores, associados à falta de acesso a informação sistemática e de qualidade e a serviços de saúde eficientes, como ocorre com a população mais carente (como os participantes deste estudo), diminuem as possibilidades de adoção de comportamentos preventivos. Essas desigualdades sociais são muito antigas em nossa sociedade e estão no cerne das vulnerabilidades específicas dessa população ao HIV/aids.

É importante investir em estratégias de prevenção entre a população jovem, como por exemplo, a realização do teste anti-HIV, pois, como observado neste estudo, apesar dos programas do Departamento de DST, Aids e Hepatites Virais de incentivo à realização do teste (Programa "Fique sabendo", por exemplo), esse comportamento ainda não faz parte do cotidiano dos jovens brasileiros.

\section{Referências}

ANTUNES, M. C. Diferenças na prevenção da aids entre homens e mulheres jovens de escolas públicas em São Paulo. Revista de Saúde Pública, São Paulo, v. 36, Supl. n. 4, p. 88-95, ago. 2002.

AYRES, J. R. C. M. et al. Vulnerabilidade e prevenção em tempos de Aids. In: BARBOSA, M. R.; PARKER, R. (Org.). Sexualidade pelo avesso: direitos, identidades e poder. Rio de Janeiro: IMS/ UERJ, 1999. p. 49-72.

BATISTA, L. E. Masculinidade, raça/cor e saúde. Ciência e Saúde Coletiva, Rio de Janeiro, v. 1o, n. 1, p. 71-8o, jan./mar. 2005.

BERTOLDO, R.; BARBARÁ, A. Representação social do namoro: a intimidade na visão dos jovens. Psico-USF, Itatiba, v. 11, n. 2, p. 229-238, jul./dez. 2006.

BOUSFIELD, A. B. Divulgação do conhecimento científico sobre aids e representações sociais. Florianópolis, 2007. Tese (Doutorado em Psicologia) - Programa de Pós-Graduação em Psicologia, Universidade Federal de Santa Catarina, 2007.
BORGES, A.L. V.; SCHOR, N. Início da vida sexual na adolescência e relações de gênero: um estudo transversal em São Paulo. Cadernos de Saúde Pública, Rio de Janeiro, v. 21, n. 2, p. 499-507, mar./ abr. 2005 .

BRASIL. Ministério da Saúde. Guia de Vigilância Epidemiológica. Brasília: Ministério da Saúde, 2005 .

BRASIL. Ministério da Saúde. Programa Nacional de DST/aids. Boletim Epidemiológico - Aids.

Brasília, 2007. Disponível em: <http://www2.aids. gov.br/data/Pages/LUMIS9A49113DPTBRIE.htm>. Acesso em: 24 abr. 2009.

BUDD, R. J. Predicting cigarette use: the need to incorporate measures of salience in the theory of reasoned action. Journal of Applied Social Psychology, New Jersey, v.16, n. 8 , p. 633-685, nov. 1986.

CAMARGO, B. V. ALCESTE: Um programa informático de análise quantitativa de dados textuais. In: MOREIRA, A. S. P.; JESUÍNO, J. C.; CAMARGO, B. V. (Org.). Perspectivas teórico-metodológicas em representações sociais. João Pessoa: UFPB, 2005, p. 511-539.

CAMARGO, B. V. Communication et prévention du sida. Études sur le rapport entre l'information télévisuelle, les représentations sociales et la pratique préventive chez les jeunes lycéens. Paris, 1997. Tese (Doutorado em Psicologia Social) - École des Hautes Etudes en Sciences Sociales, 1997.

CAMARGO, B. V.; BARBARÁ, A.; BERTOLDO, R. B. Um instrumento de medida da dimensão informática da representação social da aids. In: Jornada Internacional, 4 e Conferência Brasileira sobre Representações Sociais, 2, 2005, João Pessoa. Anais... Textos Completos da IV Jornada Internacional e II Conferência Brasileira sobre Representações Sociais, 2005, v. 1, p. 1385-1395.

CAMARGO, B. V.; BERTOLDO, R. B. Comparação da vulnerabilidade em relação ao HIV de estudantes da escola pública e particular. Estudos de Psicologia, Campinas, v. 23, n. 4, p. 369-379, out./dez. 2006. 
CAMARGO, B. V.; BOTELHO, L. J. Aids, sexualidade e atitudes de adolescentes sobre prevenção contra o HIV. Revista de Saúde Pública, São Paulo, v. 41, n. 1, p. 61-68, fev. 2007.

CAMARGO, B. V. et al. Variáveis psicossociais associadas à vulnerabilidade ao HIV/aids: estudo comparativo da população negra com a população branca. Relatório técnico de pesquisa. Brasília, Ministério da Sáude, 2007. p.1-88.

EHRHARDT, A.; WASSERHEIT, J. N. Age, gender, and sexual risk behaviors for sexually transmitted diseases in the United States. In: WASSERHEIT, J. N. et al. (Org.). Research issues in human behavior and sexually transmitted diseases in the AIDS era. Washington: American Society for Microbiology, 1991. p. 97-121.

FERREIRA, M. A. Conhecimento e percepção de risco sobre o HIV/aids: um perfil da população brasileira em 1998. Cadernos de Saúde Pública, Rio de Janeiro, v. 19, Supl. n. 2, p. 213 222, jun. 2003.

FLORIANÓPOLIS (Município). Prefeitura Municipal de Florianópolis. Dados da Vigilância Epidemiológica de Florianópolis: Sistema CTA. Florianópolis: PMF. 2009.

FOREMAN, F. E. Intimate risk: sexual risk behavior among African American college women. Journal of Black Studies, Filadélfia, v. 33, n. 5, p. 637-653, may 2003.

GHIGLIONE, R.; MATALON, B. O inquérito: teoria e prática. Oeiras: Celta, 1993.

GIACOMOZZI, A. I. Casamento e aids: uma questão de confiança. São Paulo: Mackenzie, 2005.

GIACOMOZZI, A. I.; CAMARGO, B. V. Eu confio no meu marido: estudo da representação social de mulheres com parceiro fixo sobre prevenção da aids. Psicologia: Teoria e Prática, São Paul, v. 6, n. 1, p. 31-44, jan./jun. 2004.

GODIN, H. L'éducation pour la santé: lês foudaments psychosociaux de la définition des messages éducatifs. Sciences Sociales et Santé, Paris, v. 9, n. 1, p. 67-94, mars 1991.
GORN, G. J. The effects of personal involvement, communication discrepancy, and source prestige on reactions to communications on separatism. Canadian Journal of Behavioral Science, Washington, v. 7, n. 4, p.369-386, oct. 1975.

GRIEP, R. H.; ARAÚJO, C. L. F.; BATISTA, S. M. Comportamento de risco para a infecção pelo HIV entre adolescentes atendidos em um Centro de Testagem e Aconselhamento em DST/aids no Município do Rio de Janeiro, Brasil. Epidemiologia e Serviços de Saúde, Brasília, v. 14, n. 2, p. 119-126, jun. 2005 .

GUTNIK L. A. et al. The role of emotion in decision-making: a cognitive neuroeconomic approach towards understanding sexual risk behavior. Journal of Biomedical Informatics, Oxford, v. 39, n. 6, p. 720-736, dec. 2006.

KROSNICK, J. A. The role of the attitude importance in social evaluations: a study of policy preferences, presidencial candidate evaluations, and voting behavior. Journal of Personality and Social Psychology, Washington, v. 55, n. 2, p.196210, aug. 1988.

JODELET, D. Representações do contágio e a aids. In: MADEIRA, M.; JODELET, D. (Org.). Aids e representações sociais: à busca de sentidos. Natal: UFRN, 1998. p.17-45.

LIMA, M. L. P. Atitudes. In: VALA, J.; MONTEIRO, M. B.(Org.). Psicologia social. Lisboa: Fundação Calouste Gulbenkian, 1996. p.197-199.

LOPES, F.; BUCHALLA, C. M.; AYRES, J. R. C. M. Mulheres negras e não negras e vulnerabilidade ao HIV/aids no estado de São Paulo, Brasil.

Revista de Saúde Pública, São Paulo, v. 41, supl. n. 2, p. 39-46, dez. 2007.

MADUREIRA, V. S. F.; TRENTINI, M. Da utilização do preservativo masculino à prevenção de DST/ aids. Ciência e Saúde Coletiva, Rio de Janeiro, v. 13, n. 6, p. 1807-1816, nov./dez. 2008.

MARTINS, L. B. M. et al. Fatores associados ao uso de preservativo masculino e ao conhecimento sobre DST/aids em adolescentes de escolas públicas e privadas do município de São Paulo. Cadernos de Saúde Pública, Rio de Janeiro, v. 22, n. 2, p. 315-323, fev. 2006. 
MATOS, M.; FERES-CARNEIRO, T.; JABLONSKI, B. Adolescência e relações amorosas: um estudo sobre jovens das camadas populares cariocas. Interação em Psicologia, Curitiba, v. 9, n. 1, p. 2133, jan./jun. 2005.

MCGRAW, K. M.; LODGE, M.; STROH, P. K. On-line processing in candidate evaluation: the effects of issue order, issue salience, and sophistication. Political Behavior, New York, n. 12, p. 41-58, 1990.

OLIVEIRA, S. H. S.; DIAS, M. R.; SILVA, M. I. T. Adolescentes e aids: fatores que influenciam a intenção de uso do preservativo. DST: jornal brasileiro de doenças sexualmente transmissíveis, Rio de Janeiro, v. 17, n. 1, p. 32-38, 2005.

OLTRAMARI, L. C.; CAMARGO, B. V. Representação social de mulheres profissionais do sexo sobre a aids. Estudos de Psicologia, Natal, v. 9, n. 2, p. 317323, maio/ago. 2004.

PARKER, H. A.; PERRY, R. W.; GILLESPIE, D. F. Prolegomenon to a teory of attitude-behavior relationships. Pakistan journal of psychology, Karachi, v. 7, p. 21-39, dez.1974.

REINERT, M. Une méthode d'analyse des données textuelles et une application : Aurelia de G. de Nerval. Bulletin de Méthodologie Sociologique, Paris, v. 26, p. 24-54, 1990.

RODRIGUES, A.; ASSMAR, E. M. L.; JABLONSKI, B. Psicologia social. Petrópolis: Vozes, 2002.

ROSENBERG, M. J.; HOVLAND, C. I. Cognitive, affective, and behavioural components of attitudes. In: HOVLAND, C. I.; ROSENBERG, M .J. (Org.). Attitude organisation and change: an analysis of consistency among attitude components. New Haven: Yale University Press, 1960. p.1-14.
SPSS INC. SPSS: Advanced Models 11.o. Chicago: SPSS, 2001.

VALA, J. Representações sociais e psicologia social do conhecimento cotidiano. In: VALA, J.; MONTEIRO, B. (Org.). Psicologia social. Lisboa: Fundação Calouste Gulbenkian, 2000, p. 457-502.

VAN CAMPENHOUDT, L. et al (Org.). Sexual interactions and HIV risk: new conceptual perspectives in european research. London: Taylor \& Francis, 1997.

VIDAL, E. I.; RIBEIRO, P. R. M. Algumas reflexões sobre relacionamentos afetivos e relações sexuais na adolescência. Fractal: Revista de Psicologia, Niterói, v. 3o, n. 2, p.519-532, jun./dez. 2008.

VINACCIA, S. et al. Conductas sexuales de riesgo para la infección por Vih/Sida en adolescentes colombianos. Terapia psicológica, Santiago, v. 25, n. 1, p. 39-50, jun. 2007.

VILLELA, W. Homem que é homem também pega aids? In: ARILLHA, M.; RIDENTI, G. U.; MEDRADO, B. (Org.). Homens e masculinidades: outras palavras. São Paulo: Editora 34, 1998, p. 129-42.

ZUWERINK, J. R.; DEVINE, P. G. Attitude importance and resistance to persuasion: It's not just the thought that counts. Journal or Personality and Social Psychology, Washington, v. 70, n. 5, p. 931-944, may 1996.

Recebido em: 07/10/2009

Reapresentado em: 22/03/2010

Aprovado em: 26/04/2010 